\title{
ILUSTRAXON
}

\section{Semyotika sa Pahina at sa Piraso ng Komix}

\author{
Arbeen R. Acuña \\ arbeen.acuna@gmail.com \\ University of the Philippines
}

\begin{abstract}
Panukala ng papel na ito ang paggamit ng "ilustraxon" upang mailarawan ang operasyon ng pagbibigay kahulugan sa "komix." Sa paghalaw ng "x" sa "image x text" ni WJT Mitchell (2012) at sa pagbubuo ng bagong termino, sinikap na bigyang-linaw kung paano nagiging sublasyon ang ilustraxong tinatawag na komix na sintesis ng "ilustracion" at "ilustrasyon." Gamit ang semyotika, binigyang-pagbasa sa papel na ito ang dalawang komix na literal na naglarawan sa pag-uusap ni Padre Damaso at Maria Clara sa dulo ng Noli Me Tangere (1887) ni Jose Rizal: tatlong pahina ng Nakalarawang Noli Me Tangere (1956) na isinaayos nina Clodualdo del Mundo at Pedrito Reyes at isinalarawan ni Ric Collado, at tatlong panel ng piraso (strip) na "Clara, Join the Dark Side of the Force" (2014) ni Emiliana Kampilan, na ihinambing at ipinasailalim sa semyotikong lapit ng panunuri, ayon sa apat na antas (planes) ni Alice Guillermo (2001). Mahalaga ang unang akda bilang isa sa mga unang pagsasakomiks ng susing naratibo sa nasyunalismong Pilipino at ang huli bilang makabago at intertextual na apropriasyon ng sulatin ni Rizal. Sa pagwawakas ng papel, pinalawig kung paano nagkakahawig ang ilustraxon at ang dayalohikal na tanda (sign) ni Charles Sanders Peirce-na binubuo ng representamen, interpretant, at semyotikong obheto. Mula rito, may ilang mungkahi o tala hinggil sa salin-suri (translation studies) at komix-suri (comics studies) para sa patuloy na pag-aaral ng adaptasyong komix.
\end{abstract}

\section{Abstract}

The paper proposes the term "ilustraxon" to describe the operation of meaning-making in "komix." In deriving the "x" from WJT Mitchell's "image x text" (2012) and coining a new concept, the ilustraxon called komix works as sublation or synthesis of "ilustracion" and "ilustrasyon." Two works of komix that pictured the well-known scene toward the end of Jose Rizal's Noli Me Tangere (1887), with Padre Damaso and Maria Clara's conversation, were read using semiotic analysis: three pages from Nakalarawang Noli Me Tangere (Illustrated Noli Me Tangere) (1956) arranged by Clodualdo del Mundo and Pedrito Reyes and illustrated by Ric Collado, and three panels from the strip "Clara, Join the Dark Side of the Force" (2014) by Emiliana 
Kampilan were compared and studied through Alice Guillermo's planes of semiotic analysis (2001). The first work is a key text because it is arguably one of the earliest komiks adaptations of an essential work on Philippine nationalism and the latter because it is a contemporary and intertextual appropriation of Rizal's writing. In conclusion, the similiarities of ilustraxon with Charles Sanders Peirce's dialogical sign-composed of the representamen, the interpretant, and the semiotic object-is elaborated. The paper ends with suggestions and notes that might contribute to the disciplines of translation studies and comics studies toward further studies or analyses of komix adaptations.

\section{Keywords}

Dead Balagtas, Komiks, Nationalism, Noli Me Tangere, Semiotics, Rizal

\section{About the Author}

Tilde (Arbeen R. Acuña) is an instructor and graduate student of Araling Pilipino at the Departamento ng Filipino at Panitikan ng Pilipinas, University of the Philippines. Humanities Diliman, Likhaan, Ani, Entrada, Pingkian and other journals, anthologies, and zines have published his works. His column at DavaoToday is called "Materials, for Preposterity." 
Walang humpay na dayalogo ang huntahan ng sining at ng hinaharayang bayan. Kung idinisenyo ng batas (tulad ng Rizal Law o RA 1425) ang rekisitong babasahing likhang-sining o -pampanitikan (tulad ng Noli Me Tangere o Noli), tiyak na tagpuan o interseksyon ito kung saan magkukurus ng landas ang mga usapin, isyu, o polemiko hinggil sa "bayan." Ipinakita sa mga libro ni Hau ang mahigpit na ugnayan ng panitikan at ng pagbuo ng nasyon, lalo at ipinakita sa Noli, kathang pundasyon ng pambansang komunidad ng Pilipinas (Philippine national community), ang komplexidad ng "commitment" sa mga karakter nina Ibarra, Maria Clara, at Elias (Hau, On the Subject 153). Kaya rin pinili ang eksena nina Maria Clara at Damaso sa pagsusuring papel na ito na hinggil sa Dead Balagtas (2014) ni Emiliana Kampilan at Nakalarawang Noli Me Tangere (1956) (o NNMT) na isinaayos nina Clodualdo del Mundo at Pedrito Reyes at isinalarawan ni Ric Collado. Bilang pagtangkilik sa kolektibong pagsisikap, kolektibo ring kinikilala ng papel na ito ang tatlo bilang "MRC," awtor ng naturang adaptasyon; samantala, dahil alyas o sagisag at kung tutuusin ay maituturing na "karakter" na likha ng di-kilalang awtor ang personang "Emiliana Kampilan," hindi makagagamit ng mga biograpikal na detalye upang unawain ang kanyang akda. Kung mayroon mang susing pigura ng panitikan sa set o pangkat na ito ng mga awtor at karakter na mala-awtor, si del Mundo ito at ang kanyang panahon. May pahiwatig ang mismong pagiging anonimo ng lumikha kay Kampilan sa kasalukuyang panahon lalo at sa social media lumaganap ang Dead Balagtas, na tila idinisenyo upang i-decode sa sarili nito nang may awtonomiya marahil sa personalidad ng awtor. Sa kabila ng mga salik na ito, masasabing sapat ang mga iniwang bakas ng mga may-katha upang masundan natin ang ginagayon nitong mga komikero at komikera sa kanilang mga likhang-sining at -pampanitikang nakabatay kay Rizal.

Sang-ayon kay Hau, nagiging punong-naratibo (master-narrative) ang mga nobela ni Rizal na kinakatigan o kinokontra sa pagsisikap na pag-usapan ang mga usaping panlipunan (Necessary Fictions 11). Kasunod ng pagbasa o pagbibigay-kahulugan sa punong-naratibo ay ang pagsusulat o pagbibigay-kahulugan sa pamamagitan ng mga bungang-naratibong maaaring alinman (pero hindi limitado) sa sumusunod: pagsasalin (translation, tulad ng The Lost Eden [1961] ni Leon Ma. Guerrero), pagaangkop (adaptation, tulad ng isinakomiks na bersyong NNMT), o pag-aangkin/ paghahalaw (appropriation, tulad ng Dead Balagtas). Sa salin ni Guerrero na tila naging anakronismo, natukoy ang ilang problema: nawala sa milieu ang orihinal na nobela, napalabnaw ang mga bakas ng ironya, kalkuladong siste, at satirikal na antikolonyalismo ng orihinal. Sa pagiging tuluyang Ingles ng salin, nawala ang mga baluktot na Kastila ng mga mapagpanggap na social climber na indio/a at nabura rin ang pagsisingit ng Tagalog sa pananalita ng prayleng kinatawan ng kolonyalismo (Anderson 235-262). Samantala, sa pananagalog ni Damaso sa NNMT, nabawasan ang pagkabanyaga nito, at sa pagdibuho sa bawat karakter, naitakda ang hitsura nilang nakabatay sa interpretasyon ng MRC sa isinulat ni Rizal. Gayunpaman, may 
napatingkad pa ring katangian ng textong orihinal itong isinalin at iningles, at tiyak na may naiaambag din sa pagbibigay-kahulugan sa orihinal itong mga bersyon at apropriasong tinagalog at isinakomiks.

\section{X: ILUSTRAXON AT KOMIX}

Inangkat ng mga ilustrado ang inaninag na liwanag ng karunungan mula Europa nang mabatid na nangangapa pa sa dilim ang Pilipinas sa lilim ng anino ng emperyong Kastila. Ang "enlightenment" ng mga ilustrado ang pinatutungkulan ng "ilustracion" sa papel na ito at hindi ang "La Ilustracion" ng "Madre España." Mas palasak naman ang gamit sa "ilustrasyon" na maaaring tumukoy sa iginuguhit na imahe, o sa inilalarawang halimbawa at/o iba pang karaniwang pakahulugan sa "illustration." May alingasngas sa pagitan ng ilustracion na maikakawil sa kanluraning karunungang dala ng edukasyong pormal kung saan nananampalataya ang mga ilustrado, at ng ilustrasyon na naiuugnay sa "illiteracy" o "semi-literacy" kung saan diumano lugmok ang mga indio o di-edukado; subalit problematiko ang dikotomiyang ito ng "literate" at "illiterate," lalo at iba-iba ang pamamaraan ng "pagbasa” na hindi limitado sa alpanumerikong mga tanda at sa iisang wika. Kung ang Relihiyon noong kolonyalismong Kastila ang pangunahing institusyon ng gahum, napalitan ito ng Edukasyon nang humalili bilang mga panginoon ang kolonyalismong Amerikano, kaya hindi nakapagtatakang niyurakan noong dekada singkwenta ang midyum ng komiks, ayon sa dokumentasyon ni Jurilla, bilang "diablong nagpanibagong-bihis," "basura," at "kabalbalan" (128-129). Tila sublasyon o sintesis ang ilustraxon (na siyang magiging konseptwal na balangkas ng pananaliksik) ng karunungang ilustrado (ilustracion) at ng komiks ng kadiliman (ilustrasyon).

Hinalaw ang "ilustraxon" sa "image x text" ni Mitchell (1-2) na addendum sa paggamit niya noon ng tipograpiya upang isaad, hindi ang ano o ang depinisyon nito, kundi ang paano o ang mga paraang maipapakita ng pagkakasulat ang tila dayalektikal na relasyon ng imahen at texto: "imagetext" ang sintesis ng dalawa, "image-text" ang ugnayan (relationship) at "image/text" ang alitan (rupture) (Mitchell, Picture Theory 89). Bagamat mahirap matukoy ang katangiang magbubukod sa salita (na naririnig) at sa imahe (na nakikita) dahil "visible language" o mala-imahe itong mga salitang binabasa mo sa sandaling ito; at dahil hindi pipa ang obrang La Trahison des Images (Panlilinlang ng mga Imahe) (1928-29) ni Rene Magritte na may paglalarawan sa loob ng mismong larawan: "Cest n'est pas une pipe;" at dahil hindi aktwal na naririnig ang sinasabi sa word balloon ng tauhang nakapaloob sa mga komiks. Ipinapahiwatig ng mga suliranin o tila kabalintunaang ito na hindi maipipirmi bilang absolutong "binary opposition" 
ang salita at ang imahe sapagkat maituturing ang dalawa bilang "dialectical trope" na maaaring baguhin at pansamantalang aregluhin. Maaari itong mailagom ng notasyong inimbento ni Mitchell na "vs/as"—na maaaring isalin na "kontra/bilang." Pinapakahulugan ng "salita kontra imahe" ang kaibahan, tunggalian, at tensyon sa pagitan ng mga termino; sa kabilang banda, itinatalaga naman ng "salita bilang imahe" ang potensyal nilang magsama, maglaho, at magpalitan o mag-rilyebo (“Image X Text" 2).

Pinagsanib ni Mitchell ang "VS" at "AS" upang makabuo ang pagkurus ng landas ng "V" at "A" ng kambal na "X" sa "Image X Text" upang maipakita ang "chiasmus" sa retorika at "dialectical reversal" — ang "wika ng imahe" na magbibigay ng "imahe ng wika." Dagdag sa nabanggit na interseksyon o sangandaan, isisinsin at isisiksik ni Mitchell sa "X" ang diwa ng "unknown" o "variable" sa algebra, ang mga tanda ng operasyong matematikal (multiplication $[\mathrm{x}]$ at tabinging $\mathrm{x}$ sa addition $[+]$ ), dalawang nagsasalagang "slash" $(/)$ at $(\backslash)$ na tila sibat mula magkabilang direksyon at ponema ng "eXcess" o "eXtra" o ang "unpredictable surplus" ng lokusyong "imagetext." Ilang literal na manipestasyon ng imagetext: "naratibong isinalarawan at comics, textong litrato, matulaing experimentong may tinig at larawan, komposisyong collage, at tipograpiya mismo" (graphic narratives and comics, photo texts, poetic experiments with voice and picture, collage composition, and typography itself) ("Image X Text" 1-2; aking salin). Sa pag-aaral na ito, maitutumbas sa "imahe" ang "ilustrasyon," sa "salita" ang "ilustracion," at sa "imagextext" ang "ilustraxon," bagamat may katangian ang ilustraxon na wala sa imagextext. Kung lalahok sa laro ng tipograpiya tulad ng ginagawa ni Mitchell, halos sakto at maglalapat ang "ilustracion" at "ilustrasyon" kung pagpapatungin nang may pantay na "kerning" o espasyo ang bawat letra, maliban sa dalawa. Upang mapagsama ang dalawa, maaring magsilbing "variable" ang " $x$ " na limitado ang "value" sa set na naglalaman ng "ci" o "sy": $x=\{$ ci, sy $\}$.

Sa potensyal na sarplas, dayalektika, at sintesis ng imagextext, maaaninag pa rin ang "image," " $x$," at "text," pero tila iisang entidad na lamang ang ilustracion at ilustrasyon sa ilustraxon, marahil dahil hindi gaanong bagahe ng pag-aaral na ito ang pag-aari ng akda o awtorship (o dibisyon ng paggawa?) — na isa sa mga usapin sa comics (tignan ang Uidihir). Nagpasya ring ipalagay na ang MRC ang awtor ng Nakalarawang Noli; kaya burado na halos sa ilustraxon ang pagtatangi sa image o ilustrasyon at sa text o ilustracion; dagdag pa, hindi maiiwasang pansamantalang maisantabi muna sa pag-aaral na ito ang simboliko at kultural na kapital na nakokonsentra sa mga awtor, dahil naprodyus ang mga naturang akda sa sistemang kapitalista. May labis na halaga ang mga sulatin at mga imahe ni Rizal, subalit mapapasakamay at mapakikinabangan lamang ito ng mga may antas ng proximidad sa kanyang uri: ang gitnang-uring namamagitan sa namamanginoon at nasasakupan. Susing babasahin nga lang ang mga komix dahil paraan ito ng pagakses ng mga binasagang "semi-literate" sa binasbasang babasahin (mga textong 
Rizal), kaya mahalagang masiyasat ang interbensyon ng MRC at ni Kampilan sa paghahatid ng mensaheng maaaring makabubuti o hindi sa nakararami.

Maaari ring pakinabangan ang "x" sa pagtitilad-tilad ng pagkakaiba-iba ng comics, comix, komiks at komix. Balikang muli ang kasaysayan ng midyang popular na ito sa labas at sa loob ng bansa: pinakamasaklaw at pinagtatalunan ang depinisyon ng "comics," at sa pag-aaral na ito, magsisilbi itong "umbrella" sa tatlong nalalabing termino. Noong dekada sisenta sa Amerika unang ginamit ng kabataan ang "comix" bilang pagyakap sa kulturang "sex, drugs \& rock 'n roll" at papausbong na panitikang underground (tingnan ang Danky at Kitchen; Hatfield). Habang sa Amerika itinuturing bilang "radikal" ang "comix" ng mga intelektwal kasabay ng romantisado at inaakalang kalayaan nila sa pamamahayag, niyuyurakan sa Pilipinas ng mga elitista bilang "bakya" ang "komiks"—na palalawigin mamaya. Sa pakahulugan sa loob ng papel na ito, ang mga lokal na comics na inilimbag noong "golden age" nito noong dekada singkwenta hanggang sa pagbagsak ng industriya noong dekada nobenta ang tinutukoy ng "komiks." Pagkatapos ng naturang yugto, magpoprodyus na ng "komix" para sa "niche market" o piling mamimili na namamalengke sa mga komiks convention (komikon) na nasa deerkong venue at hindi na sa bangketa; matutunghayan ang "shift" na ito mula "pang-masa” tungong "collector's item" sa kinahinatnan ng El Indio ni Francisco V. Coching, na naging pambansang alagad ng sining noong 2014, matapos ang makailang-ulit na nominasyon. Noong 1952, mumurahing seryalisadong komiks ang El Indio na kinatatampukan ng umaatikabong aksyon at paghihiganti na ihinalintulad sa Noli at Fili ni David (123-124); tinipon ang mga isyu noong 2009 at ibinebenta sa halagang dalawanlibong piso-nagtatakda ng bagong mambabasa ang "graphic novel" na malayo na sa akses ng dating mambabasa ng "komiks" (129). Ayon kay Kukkonen, dekada nobenta naging prominente ang paggamit ng terminong "graphic novel" upang pumatungkol sa comics na inililimbag bilang isang buong libro, at hindi seryalisadong "comic books" na kadalasang kinokolekta bilang trade paperbacks (ТPB); pero sa pagtatapos ng ika-2o siglo, ibinebenta ang ilang comics TPB bilang "graphic novel" para ipahiwatig na "panitikan" ito at kung gayo'y produktong kultural (84-85). Dagdag pa ni Sabin, taong 1986 hanggang 1987 naging bukambibig ang "graphic novel" nang kolektahin sa isang libro ang susing mga comics na Dark Knight, Watchmen at Maus; samantalang 1978 naman daw ang isa sa unang paggamit ng terminong ito bilang paglalarawan ni Will Eisner sa 178-pahina comics niyang A Contract with God (247). Sa Pilipinas, ang pumalit sa komiks noong dekada nobenta ay "Philippine manga" at "graphic novel" na maaaring "independent" ang produksyon o maaari ring sa pamamagitan ng malalaking palimbagan (Lent 131-132). Kung gayon, hindi pa masasabing saklaw ng naturang termino ang $N N M T$, dahil nauna ang komiks na ito kaysa sa paggamit ng termino at may sarili itong kakanyahan at kasaysayang hiwalay sa "graphic novel." Sa papel na ito, sapat na muna ang "komiks" at "komix" sa pagtalakay. 
Ginagamit ko rin ang terminong "komix" upang tukuyin ang pagiging sining at panitikan nitong may "manipestasyong kontemporaryo" tulad ng sinaad ni David (120); hindi ako gaanong makadistansya sa midyum na "komiks" tulad ng pagdistansya ni David sa "pseudo-scholarly" (122) na Komiks sa Paningin ng mga Tagakomiks (2007) nina Randy Valiente at Fermin Salvador, dahil babasahin ko rin ang komiks na NNMT bilang komix. Kumbaga, maaaring pangngalang nakabatay sa yugto o pang-uring ginagamit sa kategorisasyon ng disiplina ang "komix" sa papel na ito. Kaya "araling komix" ito, imbis na "araling komiks," dahil may mga komiks na inilimbag noong "golden age" kung kailan mayroon pang industriya (o monopolyo) na maaaring sipatin at suriin bilang komix, i.e. gawaing-sining at -panitikan (work of art \& literature), gamit ang:

\section{SEMYOTIKA: APAT NA ANTAS}

Nagkakaiba ang materyal na larawan (picture) at ang imateryal na imahe sa paraang nabibili, nananakaw, at nawawasak ang una, subalit nananatili ang huli. Ani Wittgenstein, hindi larawan ang imahe pero matutumbasan (correspond) ang imahe ng larawan. Simbolikong anyo ang mga imahe mula mga pigura hanggang mga letra, kaya maaaring saklawin ng nosyon ng "sign" ni Peirce ang lahat mula mga litrato hanggang mga marka sa algebra (Mitchell, What Do Pictures 84-85). Kung "sign" ang imahe, at sa kasong ito, ang ilustraxong nakatungtong sa konsepto ng imagextext, hindi maaaring hindi humantong ang pag-aaral sa semyotika, o ang pag-aaral ng mga tanda (sign). Upang maisagawa ang semiotic na lapit (approach), hihiramin ang metodolohiya ng pananaliksik ni Guillermo hinggil sa pagbabasa ng imahe na mayroong apat na antas ng panunuri (planes of analysis) (Images 1-16):

Sa antas ng batayang semyotiko (basic semiotic plane) maaaring idokumento ang mga biswal at pisikal na salik ng materyal na manipestasyon ng imahe, kung saan nagkakaiba na kaagad ang NNMT at "Clara, Join..."-tatlong pahina (169-171) ng librong nasasalat at nabubuklat ang una, tatluhang-panel na piraso (strip) na mahihipo lamang kung may touchscreen na gadget itong huli.

Sa tatlong pahina ng MRC, indikasyon ang mga numerong nasa itaas-kanang bahagi ng bawat panel na nagtatakda ng ayos o order ng pagbabasa-na wala sa piraso ni Kampilan na tuluyan lang at bahala na ang likas na tendensiya ng mambabasa kung paano magpapatuloy: sa comics, madalas na "inverted S" o "Z" ang kaayusan ng pagbabasang naaayon sa mga biswal na hudyat. Bunsod marahil ng produksyon (na tatalakayin muli sa ibang antas), may "shades" na mala-kahel na 
kulay ang pahina ng MRC; samantala, kulay lila naman ang sa piraso ni Kampilan kapag nag-hover o itinapat ang cursor sa ibabaw nito, at kapag inalis ang cursor sa piraso, nagiging kulay abo ang mga bahaging kulay lila. Makikita sa pahina 169 ang "Nakaraan" (tanda ng pagiging seryalisado ng anyo o format) at "Gintong Diwa ni Rizal" (tanda ng pagdidikta ng matalinghaga at sagradong "moral lesson" o pagiging didaktiko); sa ibaba naman ng piraso sa tumblr, may komento ang awtor: "Rizal started this trope, I swear. Anyway, ang di makagets, di nagbasa ng Noli o nakanood ng Starwars. Boohoo." Masasabing nagtatakda ng "linear" na pagbasa ang pahina, batay sa ipinapahiwatig ng mga nabanggit na numero ng pagkakasunud-sunod ng panel na may bilang mula 926 hanggang 941. Imbis na "word balloon," tuluyang prosa ang nagsasaad kung sino ang may sabi ng alin, tulad ng matutunghayan sa kumbensyunal na maikling kwento. Sa piraso, may "word balloon" na puti sa unang panel at may textong itim; nabaliktad sa ikalawang panel, naging itim na may textong puti-tila may kung anong sumanib at nagpabago ng tinig ni Damaso na makukumpirma sa aninong nasa kanyang mukha.

Nagwakas sa kalungkutan ng pasko ang mga pahina, samantalang facial expression naman ni Maria Clara ang nagtuldok sa piraso, para sa nakakatawang epekto (effect). Nagsimula ang piraso sa "establishing shot" upang ipakitang sa gabi naganap ang dayalago, samantalang nasa dulo ng mga pahina ang "establishing shot" ng malungkot na gabi para marahil sa dramatikong epekto.

Sa antas na ikoniko (iconic plane) naman sinisipat ang mga kalidad ng mismong imahe na nag-aambag sa kahulugan. Magkatulad ang pigura o tauhan sa pahina at sa piraso ng komix: si Padre Damaso at Maria Clara, pero magkaiba ang pagtalakay o "treatment" sa kanila bilang mga karakter ng bawat anyo o format na bunsod marahil ng magkaibang inaasahang mambabasa.

Sa pahina, kasiningang pampanitikan ang paglikha ng tensyon sa impormasyong nalalaman ng mambabasa na hindi nalalaman ni Maria Clara-ang pagiging anak niya ng prayleng iginagalang at itinuturing niyang ninong. Sa karamihan ng panel, magkasama ang dalawang tauhan, pero may isang panel na nakapokus kay Damaso (panel 931) at dalawang nakapokus kay Maria Clara (panel 930 at 935); sa mga pagpokus na ito sa mga tauhan, tila direktang kinakausap ang mambabasa. Madalas na mas nakatataas ang pigura ng prayle dahil siya ang may awtoridad bilang kinatawan ng Simbahan at bilang "ninong" o ama ni Maria Clara. Sa piraso, tumawid nang intertextual at nadagdagan ng isa pang suson ng kahulugan nang magkaroon ng reference kay Darth Vader ng Star Wars sa pamamagitan ng tinatawag na "visual pun" (termino ni Miodrag) sa nabanggit na aninong sumapi kay Damaso at nagpabago ng tinig nito; kahit walang aktwal na tunog, "naririnig" 
marahil ng mambabasa ang gumagaralgal na tinig ni Darth Vader nang baguhin ni Kampilan ang kulay ng word balloon mula puti tungong itim, at ang wika mula Filipino tungong Ingles.

Sa pagiging iisa ni Damaso at Darth Vader, nagsasanib ang kahariang kolonyal ng mga Kastila at ang emperyong intergalactic ng "Dark Side." Hindi maiwasang tingnan si Maria Clara bilang Princess Leia, lalo at itim at puti ang huling panel, may sigaw na walang tunog o naiipit at hindi maipirmi kung "nye" na reaksyon sa punchline o daing ng kabiguan. Mapapansin ding nagsasalita ang kanikaniyang Damaso at Maria Clara ng MRC at ni Kampilan sa wika ng panahon kung kailan nailimbag ang mga gawaing-sining. Matatas managalog ang Damaso ng MRC samantalang wala na rin kahit ang ortograpiyang Kastila (pansinin ang "kumbento" imbis na "convento") sa Ingliserong Damaso ni Kampilan (pansinin na mula Hollywood, pangkulturang makinarya ng Amerika, ang punchline na "I am Your Father"). Dagdag pa rito, tila wikang pambansa at konserbatibong gawi (bibigyang-patunay sa ibang antas ng pagsusuri mamaya) ang ibinabandera ng MRC, habang pasimpleng pagpaparamdam sa saklaw ng kolonyalismo mula nakaraan ng kolonyal na Pilipinas (Damaso) hanggang hinaharap (Darth Vader) ng sanlibutang intergalactic ang masisteng paalala ni Kampilan, dagdag pa marahil dito ang pagpapaalalang mestiza ang stereotype ng "babaeng Pilipina," na bunga ng sapilitang pakikipagniig (sa madaling sabi, panggagahasa) ng kolonyalista sa india.

Sa antas na kontextual (contextual plane) isinasaalang-alang ang mga aspekto ng produksyon sa milieu ng obra na apektado sa dayalohikal na ugnayan nito sa lipunan. Muli, taong 1956 noong binigyang-tibay ang Rizal Law at tinipon ang mga isyu ng NNMT upang maging "nobela." Tulak ng pagtatangi kay Rizal ng estado bilang huwarang bayani, naging mainit ang pagsisiyasat kung bakit hindi si Bonifacio. Habang nagtatalo ang mga pantas sa akademya at sa mga institusyon noong dekada singkwenta hinggil sa repormista o rebolusyonaryong pahiwatig sa mga isinulat at ginawa ni Rizal at/o ni Bonifacio, lumalawak ang saklaw ng mga isinalarawang mga naratibo: ang komiks. Sa dekadang ito ang "golden age" ng ating komiks (Lent 72), halos kasabay ng pagtaas ng estado ng pelikula bilang "industriya" (Lumbera 329), tanda ng masiglang magiging relasyon at natural na kolaborasyon ng dalawang midyang popular dahil magkakaroon ng bersyong komiks ang mga pelikula at bersyong pelikula ang mga komiks. At siyempre, kasama itong bersyong komiks ng Noli.

Nakapaloob ang naturang dekada sa saklaw ng mga taong 1935 hanggang 1955 na kalimitang pinaniniwalaang "golden age" ng comics sa Amerika (Sabin 144), bagamat may mga peryodisasyong binibiyak sa dalawa ang naturang yugto: 
"golden age" ang 1938 hanggang 1945 at "atomic age" naman ang dekada matapos ang Ikalawang Digmaang Pandaigdig (Meskin and Cook xxiii). Sa huling kalahati ng dekada singkwenta, humina ang industriya ng comics dahil nagkaroon ng panibagong dibersyon tulad ng telebisyon (Sabin 163). May pagkakapareho rin ang istorya ng pagbagsak ng industriya ng komiks sa pag-usad ng panahon-nagiba ang pampalipas-oras, nagkaroon ng censura, nakonsentra sa monopolyo ang produksyon.

Sinisi noong dekada singkwenta ang komiks bilang tagapamandila ng "illiteracy"-pinipigilan ng mumurahing ilustrasyon ang makabuluhang ilustracion (enlightenment, metaporang malimit itinutumbas sa karunungan o edukasyon) na inaakalang lulutas sa lahat ng suliranin ng nasyon. Tulad ng videogames, TV, at pelikula sa susunod na mga dekada, magiging banta ang komiks sa "opisyal" na moralidad at kultura. Tulad ng napahapyawan kanina, naitala ni Jurilla ang ilang pagkainis at pagyurak sa mumurahing midyum: noong 1951, komiks "ang diablong nagpanibagong-bihis" (the devil in new attire) ayon sa kolum ni Henry Sendaydiego, guro sa Unibersidad ng Santo Tomas (UST). Noong 1954, delikadong salarin ("important culprit") ang komiks sa pagpapaunlad ng pagiging pala-basa ("growth of reading habit") at sa produksyon ng libro, ayon sa artikulo ni Leo Cullum sa Philippine Studies. Inilarawan ng iba pang kritiko bilang "bakya," "basura," at "isang dakot ng kabalbalan" ang comic books, subalit hindi magtatagal, pag-aaralan din ito dahil sa lawak ng saklaw na "readership" nito (128-129) - at itong lawak na ito ang magiging bentahe ng midyang popular.

Kung paniniwalaan sina Abel at Madden (vii), kwalipikado sa pagiging comics ang NNMT at Dead Balagtas dahil sumailalim ang mga ito sa reproduksyon, ang huling yugto upang maging ganap na comics ang comics. Gagatungan ito ni Bredehoft nang bigyang-diin niyang walang "facsimile" o "edisyon" ang comics sapagkat walang "orihinal" at walang pagkakapareho sa aktwal na guhit at sulat ng awtor ang comics na mapapasakamay ng mambabasa, dahil ilang proseso pa ang pagdaraanan sa pagprodyus ng comics, kumpara sa reproduksyong tipograpikal (130-156). Kakatwang kaso ang NNMT, dahil may bahagi itong tipograpikal-ang "Gintong Diwa..." na nagpapahiwatig ng ugnay nito sa produksyong pinagdaraanan ng mga babasahing purong texto tulad ng nobela at ng babasahing may imagetexto tulad ng komiks. Minakinilya ang "Gintong Diwa..." samantalang guhit- at sulat-kamay ang iba pang bahagi, na katangian ng limbag na comics (comics print) na karaniwang tumatakas o tumatanggi sa bagaheng ideolohikal ng limbag na tipograpikal (typographical print). Dumaan sa assembly line na pinamamahalaan ng tao at pinoprodyus ng makina ang NNMT. Balikan natin ang nabanggit kaninang mga bagahe nito: konserbatibong paglalahad hinggil sa kalabisan ng mga prayle at wikang pambansang kawing ng "Filipinismo"-tulak ang una ng Simbahan, at ng Estado ang huli. 
Bahagi si Clodualdo del Mundo (sangkatlo ng MRC) ng Association of Publishers and Editors of Philippine Comics-Magazines (APEPCOM) na makikipagtulungan sa Catholic Laymen's Committee for Decency upang "magmungkahi" ng "Golden Code" na magdudulot ng kusang pag-censura ng mga komikero sa sarili (selfcensorship) noong 1955 (Roxas and Arevalo 55), dekada ng "golden age" ng komiks, at ng paglakas ng paggiit ng mga maimpluwensyang burukrata para sa pambansang wikang nakabatay sa Tagalog. Kung tuluyang huminto noong 1956 (muli, taong inilimbag din ang NNMT) ang lathalaing Bicolana bunsod ng pagkalugi dahil wikang Tagalog at Ingles ang naging mas mabenta, sapagkat ang naturang mga wika ang ipinapalaganap sa mga paaralan (Barbaza 93), hindi imposibleng ganito rin ang nangyari sa ibang wikang rehiyonal.

Hindi malayong nakapag-ambag sa penomenon ng pagpapalaganap ng wikang Tagalog ang malawak na sirkulasyon ng komiks (Lent 74); ang "opisyal" nitong wika ay ang partikular na Tagalog na maririnig sa mga lansangan ng Maynila (Jurilla 151). Larangan din ang mga panel ng komiks sa pagkatuto ng mga bagong bokabularyong Tagalog lalo at masikhay, halimbawa, ang Atlas Publishing sa pagtugon sa mga fan mail na nagtatanong ng kahulugan ng mga salitang Tagalog na ginagamit sa pamagat ng mga komiks (Jurilla 156). Taliwas sa mga propagandista ng sinundang siglo na kumilos sa labas ng estado, nakapaloob dito ang mga tanyag na intelektwal noong unang bahagdan ng ika-2o siglo, kaya magtutulak ang estadong pinapangasiwaan ng Estados Unidos ng "Filipinismo," nasyunalismong nakatuon lamang sa identidad at kultura habang isinasantabi ang ekonomiya at pulitika. Angkop sa proyektong imperyalista ang tambalang Amerikanisasyon at Pilipinisasyon; kumplikado ang kasaysayan ng mga intelektwal sa kanilang pagposisyon kaugnay ng karunungang Kanluranin (Mojares 493-500; mainam ding tignan ang Thomas). May ganitong tendensiya ang mga intelektwal na ibukod ang kultural na mga usaping tulad ng identidad sa ekonomiya at pulitika, mula panahon ni del Mundo hanggang sa kasalukuyan.

Kaya mahalagang dokumento ang NNMT sa pagbuo ng pambansang wika, na isa sa mga susi sa walang patid na pagwasak-at-pagbuo ng "bayan," lalo at maaaring kasangkapanin ito ng mga institusyon tulad ng Estado at Simbahan. Ito ang ilang masisipat sa mga tagisan ng kapangyarihan noong dekada singkwenta sa pamamagitan ng NNMT, at gayundin sa "Clara, Join..." dahil tila kabaliktaran ang taong 2014 ng taong 1956 sa usapin ng wika: panahon ito ng K-12 at atras-abanteng pagtanggal sa makabuluhang bilang ng mga yunit sa Filipino sa kurikulum. Sinadya man o hindi, matutunghayan sa kumbinasyong ito na nagiging mas makatotohanan ang di-kagandahang birong nakapaloob sa Darth Damaso na umaangkin kay Maria Clara bilang anak. Mula sa melo-dramatikong Damasong natutulad sa mga nagsasalita ng Tagalog noong 1956, mayroon tayong Darth Damaso ngayong mapagkalinga at mapagbiro, pero hindi inosente ang biro dahil binibigyang-diin 
nito ang pagiging "infantilized" na bansang pala-asa sa ama o ninong o tito (Uncle Sam) na patuloy na lumalapastangan sa inang bayan. At kung babasahin pa ang Brechtiang pagbasag sa "fourth wall" ng naturang panel, tila direktang kinakausap ang mambabasa at pinaaalalahanang may pyudal at kolonyal tayong relasyon kay Darth Damaso-at dapat itong mapatid.

IV

Sa antas ng halaga sinusuri ang ugnayan ng anyo (form) at nilalaman (content), o ang kasaklawan o hangganan (degree) na nabibigyang-kahulugan at -kabuluhan ng mga katangiang materyal ng anyo o signifier ang diwang konseptwal o intelektwal o emosyonal ng nilalaman o signified sa natatanging nakalarawang tanda o sign-ang gawaing-sining (work-of-art). Nagtatagpo at nalalagom ang lahat ng antas sa huling antas na ito at madalas humahantong sa kritikang panlipunan at hinaharayang bayan ang panunuri ng halaga o saysay ng isang gawaing-sining.

Nakapaloob ang parehong akda sa pagiging susing babasahin ng mga textong Rizal na binasbasan ng batas upang maharaya ang "bayan," kaya madalas kaysa hindi, may huntahan ang anumang pagsasalin o pag-aangkop ng mga textong Rizal tulad ng Noli sa "nation-building." Mahalaga na kritikal muling pag-aralan ang mga kaisipang bitbit ni Rizal, dahil sa pagtatakda ng mga silid-aralan sa pagbabasa ng mga texto, iginigiit din ng mga aparatong ito ng estado ang mga leksyong ubligadong makuha ng mga mag-aaral upang makalampas sa asignatura. Parang alkansyang unti-unting pinupuno ng barya ang utak ng mga estudyanteng inaasahang kabisahin lamang ang lahat ng ituturo. Tinawag ito ni Freire na "banking model" ng edukasyon. Kung kontrolado ang magiging "lehitimong pagbasa" kay Rizal at iaayon lamang ito sa kurikulum ng eskwelang komersyal, maaari itong angkinin ng mga namumuhunang naghaharing uri upang simbolikong monopolyohin ang diskurso ng pagka-bansa/nacion/bayan para sa konserbatibo nilang interes bilang mga tagapamagitan ng Emperyo at ng mga mala-kolonya. Dahil tila malapit sa kahalagahan ng imahe ni Kristo sa kulturang Pilipino, ang manipulasyon sa imahe at sa bihis ni Rizal ay makapaglilingkod sa iba't ibang adyenda-sa kasong ito, adyendang neoliberal ng sistemang edukasyong idinisenyo ng estado. Mapakikinabangan ang mga texto at imaheng Rizal sa pagtatangkang maisemento ang makauring interes ng iilang komprador at mga kasapakat nila sa pamamahalang mala-pyudal at neo-kolonyal-o kontrahin ito, sa kabilang banda.

Maisasagawa lamang ang pagtunggaling ito kontra sa dominanteng pagharaya kay Rizal at sa nacion kung "patutunugin" (at/o pakikinggan?) ang mga ito sa pakahulugan ng pagbasa ni Mitchell kina Nietzsche at Marx na kapwa hindi sangayon sa pagwasak sa mga "idolo" o "fetish" na ito ng kaisipan man o ng kalipunan o ng pamilihan, dahil mas makabuluhang "patunugin" ("to sound" o "to sound-out") 
ang mga ito gamit ang "tuning fork" ng wika upang maaninag ang isang "likas na kasaysayan ng imahe na nakakawing sa moda ng produksyon at reflexes nilang ideolohikal" (Mitchell, What Do Pictures 95; aking salin). Masusubukan ang "pagpapatunog" na ito sa imahen ni Rizal at sa pag-imahen kay Rizal sa pagbasa at pagbabalik-basa (re-reading) sa kasaysayan sa panitikan at sa panitikan sa kasaysayan.

Sa inabutang kurikulum ng sekundaryang edukasyon ng aking henerasyon, apat ang rekisitong babasahin sa bawat yugto ng high school: Ibong Adarna (isinulat ng anonimong awtor na tinatayang mula Europa), Florante at Laura ni Francisco Baltazar (o "Balagtas"), Noli at El Filibusterismo (Fili) ni Jose Rizal. Patulang salaysay ang unang dalawa, samantalang salin ng mga nobelang susing babasahin sa ating panitikan at kasaysayan ang huling dalawa. Inaasahang binhi ang mga akda ng diwang makabayan, ayon sa pakahulugan ng sistemang edukasyong malakolonyal na nagtatampok sa mga ilustrado bilang huwarang mamamayan at sa malamlam na ilustracion (enlightenment) bilang gabay sa kaunlaran (development) at "nation-building." Kaya ipinagbubunyi ni Rizal tuwing buwan ng Agosto ang pambansang wika, habang kinukundena ang mga malalansang isda; samantala, laging kakabit ng islogang "kabataan ang pag-asa ng bayan" ang simplistikong pagakalang edukasyong pormal ang tanging susi sa naturang pag-asa. Samakatwid, kahit walang konsepto ng wikang "Filipino" noong kolonyalismong Espanyol at kahit ang kabiguang natamo ng ideyalismo ni Ibarra sa pananalig sa edukasyong pormal ang nagtulak sa transpormasyon tungong Simoun, ipinawiwika kay Rizal ng Departamento ng Edukasyon na karunungan ang panacea sa kanser ng lipunan.

Hindi taliwas sa nasyunalistang proyekto ng Estado at Simbahan ang adaptasyon ng MRC. Sa pagiging didaktiko ng NNMT (hudyat nito ang bahaging "Gintong Diwa ni Rizal" na para bang nagsasabing ito ang "values" na dapat makuha sa isyung ito ng komiks) may higit pang mahihinuha bukod sa tinuran ni Lent na pagiging "classic" na mala-Amorsolong mga dibuho at mainam ng timpla ng "literary" at "popular" (78). Batay sa naunang mga antas ng semyotikong panunuri, saksi ng kanyang panahon ang NNMT, sa pagiging lulan man ng anyo o estetika (anatomiyang "wasto," tiyak na kaayusan o order ng pagbabasa, at iba pang katangiang natatangi sa pagdidibuho sa yugtong kinairalan ng komiks) at ng nilalaman o mga usaping panlipunan (ang Golden Code at wikang pambansang natalakay kanina), kaya kahit tila alinsunod pa sa malakolonyal na kaisipan ang namayani sa komiks ng MRC, mahalagang akda itong dapat pagtuunan ng pag-aaral.

Dahil kasangga ng APEPCOM ang Katolisismo laban sa "imoralidad" ng pornograpiya o bomba komiks, tila naging mabait sa prayle ang MRC, lalo at maaaring mayor ang tungkulin sa pamamahala ni del Mundo sa pagsasakomiks ng Noli. Sa himig ng pa-kanang tadhanang maka-bansa ng Bagong Lipunan, 
bubuhayin ang APEPCOM bilang Kapisanan ng mga Publisista at mga Patnugot ng mga Komiks-magasin sa Pilipino (KPPKP) na siyang muling magpapatibay ng Golden Code matapos ang deklarasyon ng Batas Militar noong 1972 (Roxas and Arevalo 55); at magkakaroon ng kasong isasampa laban sa bomba komiks (58); hindi ito nalalayo sa nagtulak ng pagkakaroon ng underground comix sa America: ang Comics Code (Hatfield 11). Mahihinuha ritong hindi naman palaging agrabyado o nasa mga laylayan (margins) ang komiks-depende sa nilalaman at nagpahintulot ng nilalaman ang pagiging progresibo o reaksyunaryo ng komiks. Maaaring gamitin ito ng mga umaastang awtoridad. Ang anti-imperyalistang kritika nina Dorfman at Mattelart kontra sa Disney Comics na ipinalalaganap bilang kasangkapan ng gahum sa Latin Amerika ang isang maituturing na pangunahing araling comics na nagpakita ng pantasyang burgis, ugnayan ng midyang popular at emperyo, naturalisasyon nito sa mga stereotype ng lahi at uri. Kaya hindi absolutong "alternatibo" o marhinalisado ang comics dahil ginagamit ito sa manwal ng mga operasyon ng sandatahan ng Estados Unidos at ipinamumudmod ng CIA upang maghasik ng lagim at magtulak kaguluhan sa mga bansang hindi nila kursunada (Ellis xii).

Sa ating bansa, madalas ring ipamudmod ng mga politiko ang komiks upang pabanguhin ang kanilang imahe at tapunan ng tae ang mga kalaban sa pulitika, higit noong dekada ng Batas Militar. Kaya nabago rin ang ihip ng hangin hinggil sa pagtingin sa komiks sa pagsapit ng dekada sisenta at sitenta dahil ito ang paboritong babasahin ng masa na marapat maging malay at mulat sa Batas Militar (at kung gayo'y larangan ng negosasyon ang komiks ng ideya ng "bayan" ng masa). Kakasangkapanin din ni Marcos ang komiks sa pagkatha niya ng mito ng kaunlarang dala ng kanyang pamamahala (Lent 85). Kabilang ang komiks sa kumontra sa naturang delusyon ng iniimaheng kadakilaan, kaya kasama ito sa mga yumabong na sining ng protesta at rebolusyon. Tinutugunan at tinutunggali ang mga natalakay na reaksyunaryong opensiba ng mga progresibong sining. Halimbawa, obheto ng satire ng mga editorial cartoon ang mga Amerikanong kolonyalista at mga latak ng emperyong Kastila; makikita ang pagkutyang ito sa Lipang Kalabaw, The Independent at Philippines Free Press noong pagpasok ng ika-2o siglo (Guillermo, Protest 18). Tulad ng mga cartoon, karatula, poster, at iba pang anyong popular ang komiks kaya maaari itong maging lunsaran ng mga sining ng protesta o mga ideyang progresibo o rebolusyonaryo. Kailangang patampukin sa mga anyong popular ang makabuluhang nilalaman upang maiwaksi ang kababawan ng materyal na kadalasang ikinakabit sa naturang mga anyo. Upang maisagawa ito, kailangan ang popularisasyon at pagtataas ng pamantayan. Mainam magpakadalubhasa sa pag-aaral at pagsusuri sa mga akdang popularisado upang matuklasan kung paano pa iaangat ang pamantayang estetiko (at pampanitikan), pero hindi ito nangangahulugan ng pagpapaloob sa itinakdang pamantayan ng 
burgis na sining, ani Mao Zedong, kundi patungo sa sosyalistang sining ng bayan (people's socialist art) (Guillermo, Protest 38-39).

Hinamon ni Soledad Reyes ang klasipikasyon ng komiks sa akademya bilang "ilehitimong panitikan" at idineklara itong "salamin ng buhay" ng masang Pilipino; imbis na "pagtakas sa riyalidad," ihinaharap ng komiks ang kahirapan sa mambabasa at nabibigyang-tinig ang kanilang protesta sa mga pambihirang lakas at mapanuyang tawa ng mga karakter na may antas ng subersyon (Reyes 3-16; 160-169). Pero mainam bigyang-diing may limitasyon itong inaakalang subersibong "komedyang kritikal" na katumbas marahil ng "pamumusong" o "diwa ng mapaglarong panunuligsa o espiritu ng karnibalisasyon" (Tiongson 305). Tila nasa mababang saray ng lipunan ang pusong-walang anumang aktwal o simbolikong kapital, maliban sa siste at diskarte, bagamat hindi rekisitong maging pusong upang magkaroon ng diwang pusong (325). Kung ang pamumusong daw ay walang batayang ideolohikal dahil rekisito ang "laya" na kutyain ang alinmang ideolohiya (331) at tuligsain ang anumang hirarkiya (332), limitado kung gayon ang subersibong halakhak nito, kung isasakonteksto sa karnabal ni Bakhtin; may hanggahan ang paghalakhak lang sa suliraning panlipunan at mga salaring dapat singilin. Kung gagamitin ang pamumusong at hahalaw sa diskusyon ni McGowan hinggil sa pagpaling o pag-aalangan ng komedya tungong konserbatibo o kritikal, heto ang isang ilustrasyon: sakaling maisahan ang prayle o ang presidente dahil napilitan silang magsuot ng daster, halimbawa, at iparada ang katawa-tawa nilang itsura (may implikasyong ideolohikal maging ang pagiging katawa-tawang ito), subalit may awtoridad pa rin sila sa kabila ng pagtatanghal nila bilang ulol sa publiko nila, lalong napagtitibay ang kapangyarihan nilang nangingibabaw pa rin sa mga nagsisitawanan. Naging obheto man saglit ng komedya, sila pa rin ang naghahari at nasa kanila ang huling halakhak. Maaaring umusbong ang komedyang kritikal kung lalampas ito sa anumang "pinahintulutan" ng prayle o presidente (208) - at kung ang naturang mga pigura ng awtoridad mismo ang mapipikon at magiging tunay na obheto ng katawanan, tulad ng pinalawig ni Guillermo hinggil sa sining ng protesta at rebolusyon.

Humantong sa puntong mapapaloob na sa institusyon ng estado at mapapabilang na ang mga ilustrador ng komiks sa mga exhibit ng Cultural Center of the Philippines, makikilala silang mga beteranong dibuhista ng Marvel Comics at DC Comics sa Amerika at mababansagan ang komiks na "ating pambansang "libro"' ("our national 'book") ni Clodualdo del Mundo-may pahiwatig ang panipi niya ng duda sa pagiging lehitimong babasahin ng komiks (Jurilla 129-131). Isa sa nakikilalang likha ni del Mundo ang NNMT (Roxas and Arevalo 68). Paisa-isa munang inilabas (serialized) ang mga isyu ng naturang komiks hanggang tipunin ito noong 1956 ng Capitol Publishing House sa iisang pabalat kaya maituturing ang isinalarawang nobela ng unang Pilipino bilang potensyal na prototipo ng graphic 
novel sa ating bansa, pero sa salungatan ng komiks ng kadiliman at textbook ng karunungan sa kontexto ng kasaysayan ng aklat sa Pilipinas, nakukumplika ang ironya ng pagiging nobelang nakalarawan ng adaptasyon ng MRC — na hindi mawari kung sopistikadong panitikang kawili-wili o "cheap" na libangang pampanitikan. Sa pagtingin ni Art Spiegelman, awtor ng autobiograpikal na graphic novel na Maus (1986) hinggil sa Holocaust, tila "hindi maililigtas ng aspirasyon ng comics na matangkilik bilang sining at panitikan sa pagiging ganap nitong anakronismo" lalo at tila nagbago ang reputasyon nito mula "icon of illiteracy" tungong "last bastions of literacy" dahil hindi na pinagtitiyagaan ang pag-decode sa mga ito (Rosenkranz 27; aking salin).

Kung muling babalikan ang kasaysayan ng kolonyalismo, ang Simbahan bilang institusyon ang maituturing na pangunahing aparatong ideolohikal ng estadong kolonyal sa ilalim ng mga Espanyol, samantalang ang Paaralan naman ang sa mga Amerikano. Kaya, tulad ng nabanggit sa unang bahagi ng artikulong ito, binansagan sa kolum ni Sendaydiego (guro sa UST) noong 1951 na "diablong nagpanibagongbihis" ang komiks. Ang "demolisyon" (o "arson" para mas maging angkop sa metapora ng diablo at apoy ng impyerno?) sa reputasyon ng komiks bilang anyo ay nagatungan pa ni Cullum sa Philippine Studies journal (na pinamamahalaan o pinamamatnugutan ng ADMU) noong 1954. Nabibigyang-katuwiran kung gayon ang pagiging bagong Relihiyon ng Edukasyon, kung saan tila katumbas ang mga prayle ng mga intelektwal na may dismaya pa noon sa komiks bilang anyo. Kumpara sa panahon ng kolonyalismong Amerikano, sa yugto naman ng kasaysayan kung kailan nailimbag ang Noli at Fili, hindi gaanong naging usapin para sa mga awtoridad ang nobela bilang anyo, kundi kung ano ang nakapaloob sa anyo: ang nilalamang heretiko at subersibo, para sa Simbahang kumakasangkapan sa pamahalaang kolonyal sa pagparusa sa mga may kopya ng mga nobela.

Samantala, sa 1956, kumplikado na at masalimuot ang debate hinggil sa Noli at Fili dahil hindi na ganoong kalakas ang Simbahan, kumpara noong panahon ng kolonyalismong Espanyol. Kaya may naglakas-loob maghain ng Senate Bill (SB) 438 at ng House Bill (HB) 5561. Nagkaroon ng papel ang Simbahang Katoliko sa pagkontra sa SB 438 na ihinain ng mga senador na sina Claro M. Recto at Jose P. Laurel upang gawing "compulsory reading" ang dalawang obra ni Rizal, ang HB 5561 naman ang katumbas nito sa Kongreso. Ilan sa naging argumentong nakaangkla sa relasyon sa Simbahan ng mga kontra sa HB 5561 ay ang kabawasan sa boto sa susunod na eleksyon dahil marami ang Katoliko sa mga botante sa distrito at ang pagpunta sa purgatoryo ng mga maka-Rizal (Iniego 158). Samantala, sa Senado, matindi ang pagbatikos ng hirarkiya ng Simbahang Katoliko hanggang sa puntong binilang nila ang mga pahinang may anti-Katolisismo at patriotismo, kung saan ang una ang nakalalamang. Ang iskor, sa 333-pahinang Noli: $120>25$ at sa 293-pahinang Fili: 80 > 41 (157). Nilagdaan ni Presidente Ramon Magsaysay at naisabatas ang RA 
1425 noong Hunyo 12, sa kompromisong hindi magiging "compulsory reading" ang mga nobela, hindi kaagad isasama sa lahat ng kurikulum ng paaralan, batayang teksto lamang ang "unexpurgated version," at mayroong "exemption" kung may sinumpaang salaysay na isusumite ang estudyantang nagsasaad na kontra sa paniniwala niya ang kursong Rizal (Iniego 162-163).

Hindi gaanong magiging problema ng NNMT ang pagtuligsa mula sa Simbahan, dahil katuwang ng APEPCOM (na, muli, minsang pinamunuan ni Del Mundo, isa sa nagsaayos ng adaptasyong komiks) ang isang institusyong Katoliko sa krusada para sa kabutihang asal na may paggalang diumano sa lahat ng paniniwala, lalo nga at detalyado ang gabay nitong "Golden Code" na may iba-iba pang seksyon. Sa ilalim ng seksyong "Religion" nakasaad na: 1) hindi kailanman maaaring payagan ang pag-atake sa anumang sekta o relihiyon, bagamat maaaring ipakita ang mga abusong nagkukubli sa ngalan ng relihiyon; 2) hindi pwedeng ilarawan ang mga pari o anumang ministro ng mga sekta sa mga kalagayang malaswa o katawatawa, maliban kung kinakailangan sa pagpapahayag ng katotohanan; at 3) dapat iparamdam sa mambabasa ang kahalagahan ng pananampalataya dahil ang bawat kinikilos at iniisip ng bawat tao ay nakikita ng Panginoong Maykapal, na siyang gumagabay sa tadhana ng bawat isa (aking malayang salin mula sa orihinal na Ingles na nasa Roxas at Arevalo 56).

Bagamat wala pang natatagpuang opisyal na polisiya ang mananaliksik, hindi imposibleng itinuring bilang katumbas ng Noli ang NNMT, upang maituro pa rin ang aral at buhay ng pambansang bayani at ng kaakibat niyang nasyunalismong opisyal. Sa puntong ito, wala pa ring nahahanap ang mananaliksik na kopya o banggit na nagkaroon ng sequel na Nakalarawang Fili. Naging matagumpay man o hindi, nagkaroon ng oportunidad ang Simbahan, sa pakikipagtulungan ng APEPCOM, upang humubog ng sariling bersyon nila ng Rizal. Sa isang banda, hindi malayo sa hinagap na nabigyan ng pagkakataon ang hirarkiyang Katoliko na mapaamo ang nilalaman ng ereheng obra maestra at ang anyo ng demonyong nagpanibagong-bihis. Sa NNMT, nagkompromiso at nagtagpo ang interes ng mga mambabatas (Estado) at ng mga sugo ng Panginoon (Simbahan), kaya mahalaga itong dokumento sa isang yugto ng nasyunalimsong Pilipino noong 1956.

Tugon itong panunuring komix sa pagsisikap na mabigyang-kabuluhan ang atras-abanteng reputasyon ng komix sa kani-kanyang milieu. Hindi [pa] hinarap ni Kampilan ang mga suliranin at usapin ng MRC, dahil walang pablisyer na mamomroblema sa kikitain at sa kuneksyon sa mga institusyong tulad ng Simbahan at Estado. O maaring mas mainam sabihing hindi pa hinog ang panahon upang matantsa ang halaga at saysay ng Dead Balagtas (at inilathala ng Anino Comics ngayong taon ang unang tomo: Dead Balagtas: Mga Sayaw ng Lupat at Dagat; may pahapyaw na suri sa Acuna). Ang naturang kuneksyon sa mga institusyon ang 
malamang na naging dahilan kung bakit nalagpasan ng NNMT ang halos kalahating siglo tungo sa ating panahon, kaya hindi rin matatawaran ang kahalagahan nito upang maunawaan ang nakaraan at mapakinabangan sa kasalukuyang yugto tungo sa inaasahang mas mainam na kinabukasan. Panahon ang isang makasusukat sa kahusayan ng likhang-sining, at tunay ngang maaga pa upang tasahin ang halaga ng Dead Balagtas, pero sa talas ng kritika, sa kabila ng kaiklian, nakikinitang magiging kasing-halaga ito ng NNMT, kunsakaling hindi ito mababaon sa limot (na mukhang hindi naman magkakaganoon), tulad ng muntik nang mangyari sa gawa ng MRC.

\section{SEMEIOSIS: DAYALOHIKAL NA TANDA}



Fig. 1. Pinakalaganap na litrato ni Rizal

Matapos suriin ang pahina ng MRC at piraso ni Kampilan, ilalarawan sa bahaging ito kung paano gumagana ang kanilang mga akda bilang tanda at kung paano nagkakaroon ng mga pagbasa. Kung ginamit ang semyolohiyang Sausurrian sa panunuri, gagamitin ang semeiosis na Peircian para lamang sa paglalarawan. Tinatawag ang tanda (sign) ni Peirce na "representamen" na, aniya, ay "kung-anong nangungusap sa kungsino hinggil sa kung-ano sa humigit-kumulang na kapasidad o pagsasaalang-alang" (something which stands to somebody for something in some respect or capacity; aking tangka ang salin). Sa isip ng nakakikita ng representamen, nabubuo ang katulad, kung hindi man mas maunlad, na tanda na tinawag niyang "interpretant" at kinakatawan nito o tumitindig ito para sa ("stands for") isang bagay na tinawag niyang "object" (15). Kung maaaring magpalitpalit ang bawat sangkap ng tanda upang maging ang isa sa dalawa pang sangkap (Merrell 29) at kung mga tanda rin maging ang mga ideya (Eco 24), walang wakas samakatwid ang pagbibigay-kahulugan sa tanda.

Halaw sa halimbawa ni Merrell, kung litrato ni Rizal (palagay ko, magkahawig ang naiisip nating imahe dahil wala naman yatang ibang laganap na photo si Rizal, pero para matiyak, tignan ang imahe sa itaas) ang representamen at ang dibuho ng tauhan niyang si Ibarra sa komiks ng MRC ang semyotikong object, ang interpretant ang ugnayan nilang dalawa at sa puntong ito, bansagan nating konsepto $n g$ pagkakahawig $n g$ dalawa-isang interpetant na maaaring humantong sa pagiging bagong representamen ay ang konsepto ng pagiging iisa ni Ibarra at ni Rizal, na may karampatang semyotikong obheto na pampublikong paaralan; panibagong 




interpretant ang konsepto ni Ibarra / Rizal ng pedagohiya. Kung termino sa konsepto ng ilustraxon ang gagamitin, litrato ni Rizal ang ilustracion, dibuho ni Ibarra ang ilustrasyon at konsepto ng pagiging iisa o pagiging autobiograpikal na alter-ego ng isa't isa ang ilustraxon. Maaaring magrilyebo ng tungkulin ang tatlong salik. Ang alter-ego na ilustraxon ay magiging ilustracion / ilustrasyon na may karampatang ilustrasyon / ilustracion na pampublikong paaralan. Pedagohiya ni Rizal / Ibarra ang ilustraxon. Kinumpirma sa sekundaryong mga sangguniang nagbigay-kahulugan (interpret) sa teorya ni Peirce hinggil sa tanda ang paunang pagkakaunawa ritong hahantong sa "unlimited semiosis," bagamat ilalaglag din niya ang ideyang ito sa huli niyang mga sulatin (68-72; Potts 22; Atkin). Kung tanda ang bawat interpretant, nagbubuhat ang "kaisipang dayalohikal" sa konsepto ni Peirce ng magkakasunod na interpretant (tignan ang dayagram sa itaas mula kay Chandler 32-33).

Kung tama ang pagkakaunawa sa Peircian na tanda at sa bumubuo ritong / $/ \mathrm{o}$ representamen (signifier / simbolo o anyo ng tanda), /i/ o interpretant (signified / diwa [sense] ng tanda / sign-vehicle?), at /so/ semyotikong object (referent / kinakatawan ng tanda [what the sign stands for]), maaaring ilatag ang suliranin ng pananaliksik na ito (at kinapapaloobang mas malawak na tesis) sa gayong mga termino: Kung konsepto ng pagka-pambansang bayani ni Rizal ang / $/ \mathrm{r} /$ at Noli Me Tangere ang /so/ nito, ang ideya ng pagiging rekisitong babasahin ng Noli sa buong bayan ang /i/ na siyang magiging /r/ng / so/ na Rizal Law. Ang /i/ng huling tambalan ay ang tungkulin ng huwarang mamamayan na basahin ang naturang rekisito. Magiging / $\mathrm{r} /$ muli ang tungkuling ito na may /so/ na "illiteracy" ng karamihan ng mamamayang dapat gampanan ang naturang tungkulin. Ang /i/ na 
magiging panibagong / $/ \mathrm{r}$ ay ang pangangailangang mabasa ng mga "semi-literate" ang bersyon ng Noli na may /so/ na komiks. Hahantong na sa wakas sa /i/ at /r/ na NNMT na may /so/ na Noli at, dito na marahil tatapusin ang kawalang-katapusang ito: may interpretant na pag-aaral na "Dayalektika ng Kaliwanagan: Ilustraxon at Ang Nakalarawang Noli" (na maaaring maging representameng kinakatawan ng kung anong semyotikong object na magbibigay-daan muli sa panibagong interpretant; kung paatras, ang sinimulang representameng konsepto ng pagkapambansang bayani ni Rizal ay interpretant ng naunang tambalan ng object at tanda). Sa ibang salita, ilustracion ang Noli (1887) ni Rizal na may karampatang ilustrasyong NNMT (1956) ng MRC na ang sublasyon o sintesis ay ang ilustraxong Dayalektika ng Kaliwanagan (2018?), na siyang tentatibong pamagat ng aking gradwadong tesis.

Samakatwid, itong pag-aaral ay batay sa pagkakaunawa ko (tulak ng kinaiiralan kong posisyunalidad at sirkumstasyang panlipunan) sa $N N M T$ na batay sa pagkakaunawa ng MRC (tulak ng kinaiiralan nilang posisyunalidad at sirkumstansyang panlipunan) sa Noli Me Tangere na batay sa pagkakaunawa ni Rizal sa mga isyu ng kanyang panahon; bawat pagkakaunawa ay apektado at nakaaapekto sa nagpapatuloy na pagharaya ng "bayan." Nagkakaroon ng sarplas ang kaisipan ni Rizal dahil nakaangkla sa kanyang imahen ang (pag-)imahen ng nacion; nagsimula marahil maging tanda sa representameng Noli ang huntahan hinggil sa naturang pag-imahen sa nacion. Kung gagamitin ang kategorisasyon ni Peirce, maituturing na tanda maging ang mga salita dahil mga linguistikong tanda itong pinagkasunduan sa "social convention" kaya maaaring maging sangkap ng tanda maging ang mga abstraksyon o konsepto (tulad ng ipinakitang halimbawa sa naunang talata) - "simbolo" ang kategorya nito. Tinatawag naman niyang "icon"

ang tanda na may "resemblance" sa kinakatawan nito, gaya ng Ibarrang dibuhong katha ng MRC na tila halaw sa kaisa-isang litrato ni Rizal at marahil ang NNMT sa mismong Noli; samantala "index" ang pananaliksik na ito sa nabanggit na adaptasyon ng MRC at sa orihinal ni Rizal, usok itong papel ng dalawang apoy.

\section{TUNGUHIN NG ILUSTRAXON AT ADAPTASYONG KOMIX}

Panimulang pag-aaral pa lamang ito, at tulad ng naipahiwatig, patungo ito sa mas masaklaw na tesis. May ilang mahalagang tala ang tagubilin o paalalang hudyat mula sa "Towards a Theory of Comic Adaptation," MA thesis ni Beineke na nais ding bigyang-diin ng papel na ito: ang pagpuna sa tendensiyang magbabad lamang sa pagsusuri sa katapatan ng adaptasyon sa orihinal (fidelity review), sa paggawa ng sarbey ng mga adaptasyon ng pampanitikang tekstong tinatalakay (survey article), 
at sa pagdisenyo ng paraan upang maiangkop sa silid-aralan ang adaptasyon (pedagogical article).

Habang tinatangkilik ang kahalagahan ng orihinal at ang identipikasyon dito ng adaptasyon, marapat ding ituring na may kakanyahan ang akdang deribatibo; ani Hutcheon, maraming bersyon na umiiral "laterally" at hindi "vertically" (xiii), kaya kapwa nagtutulungan ang orihinal at ang adaptasyon upang mabigyang-paliwanag ang isa't isa (maaari ring tignan ang aking salin-suri sa tulang "Kawayan" ni Bomen Guillermo sa Acuna bilang suhay sa puntong ito). Dahil hindi produktibong pagaralan ang mga akda nang magkahiwalay (Beineke 8-11), muling babalikan ang pinaghalawan ng MRC at ni Kampilan. Ani Hutcheon, nagaganap ang "recoding" dahil tungo sa ibang midyum ang adaptasyong kadalasang may operasyon ng pagbabawas (subtraction) o pagpapaikli (contraction), at samakatwid, pagtistis (surgery) (12). Kung gayo'y may "re-mediation" o "partikular na mga pagsasalin sa anyo ng transposisyong intersemiotic mula isang sistema ng tanda (sign) (tulad ng mga salita) tungo sa isa pa (tulad ng mga imahe) - [...] tulad sa transmutasyon o transcoding, kung saan kailangan ang re-coding tungo sa isang bagong pangkat ng mga pagkakasunduan at mga tanda" (specifically, translations in the form of intersemiotic transpositions from one sign system (for example, words) to another (for example, images) - [...] as in transmutation or transcoding, that is, as necessarily recoding into a new set of conventions as well as signs) (16; aking salin). Masalimuot at kumplikado ang partikular na adaptasyong ito ng Noli, na nilalayong balikan ng mananaliksik na ito para sa mas komprehensibong pananaliksik.

Kung gayon, kailangang tignan sa dalawang antas ang pagsasalin. Sa pagpapakahulugan kay McFarlane ni Beineke, pinagbubukod kung ano ang inililipat mula sa orihinal na nobela (ito ang naratibo) at kung paano ang ginagawang paglipat (ito ang midyum, o "mode of enunciation" o "system of signification") (16-17). Malamang na dagdag-bawas ang kahinatnan ng paglilipat dahil bukod sa mapakikinabangang aparato (available tools) para sa pagkatha at pagbuo (na tinalakay rin ni Beineke), nagkakaiba rin sa paraan ng pagbasa ang pinagkunang nobela at pinaglipatang comics, dahil "linear" ang pagbabasa sa una samantalang walang itinatakdang "wasto" at iisang pamamaraan ng pagbabasa sa huli, bagamat mabibigyang-gabay ng awtor ang mambabasa sa pamamagitan ng itsura ng teksto (19-20) at magsisilbi ring gabay ang mga palatandaang biswal, tulad halimbawa ng mga "deictic gaze" o titig ng mga karakter, prominenteng mga linyang tawagpansin, direksyon ng mga dayalogo, at iba pa (Kukkonen 18). Tulad ng nabanggit, tila kumbensyon sa panahon ng NNMT ang bilang sa panel, kaya sa isang banda, tila may iminumungkahing lapit (approach) na "wasto" at "linear" na pagpapatuloy sa pagbabasa; walang ganitong katangian ang "Clara, Join..." at mas nakabatay sa mga nabanggit na biswal na hudyat. Tulad ng natunghayan sa semyotikong panunuri, nag-aambag sa kahulugan at sa nilalaman ang mga detalye ng anyo. 
Bunsod ng mga potensyal na teorya at pamamaraan, inaasahang makakapagaambag din angkabuuan ng kinapapaloobangtesis ng papel na ito kungsaan magiging bahagi ang pag-aaral na ito sa metodolohiya lalo at may natatanging suliranin at dagdag na salimuot ang adaptasyon mula magkaibang sistema ng gramatika at bokabularyo tungo sa isa pa, mula Kastilang Noli tungong Nakalarawang Noli sa yugto ng kasaysayang papalakas ang institusyonalisasyon ng estado sa wikang pambansa. Sa puntong ito, napahapyawan sa isang eksena kung papaano gagamitin ang semyolohiyang Saussurian na inangkop ni Guillermo at kung paano inilarawan ng semeiosis na Peircian ang proseso ng pagbubuo ng kahulugan sa piling (at susing) bahagi ng NNMT, at sa pagtalakay ng Dead Balagtas sa parehong eksenang unang inakda ng pambansang bayani. Sa parehong halimbawa, naipakita ang potensyal ng pahina (comics page) at piraso (comics strip) sa pagbubuo ng saysay sa kasaysayan at sa panitikan. 


\section{APENDISE}

\section{Mula sa Dead Balagtas}


"Clara, Join the Dark Side of the Force." Tatlong panel mula sa Dead Balagtas webcomics ni Emiliana Kampilan, 2014. Simple lang ang istruktura ng piraso o strip: Dayalogo sa una, kung saan makikita ang naguusap na karakter. Monologo sa ikalawa, kung saan sinaniban ng intergalaktikong puwersa ang prayle at naging Darth Damaso. Tahimik na sigaw sa ikatlo, na maaaring magsilbing espasyo upang pagmunian ang birong may bagahe, na tila may iba pang nais ipasaliksik at ipaunawa sa mambabasa. 


\section{Mula sa Nakalarawang Noli}

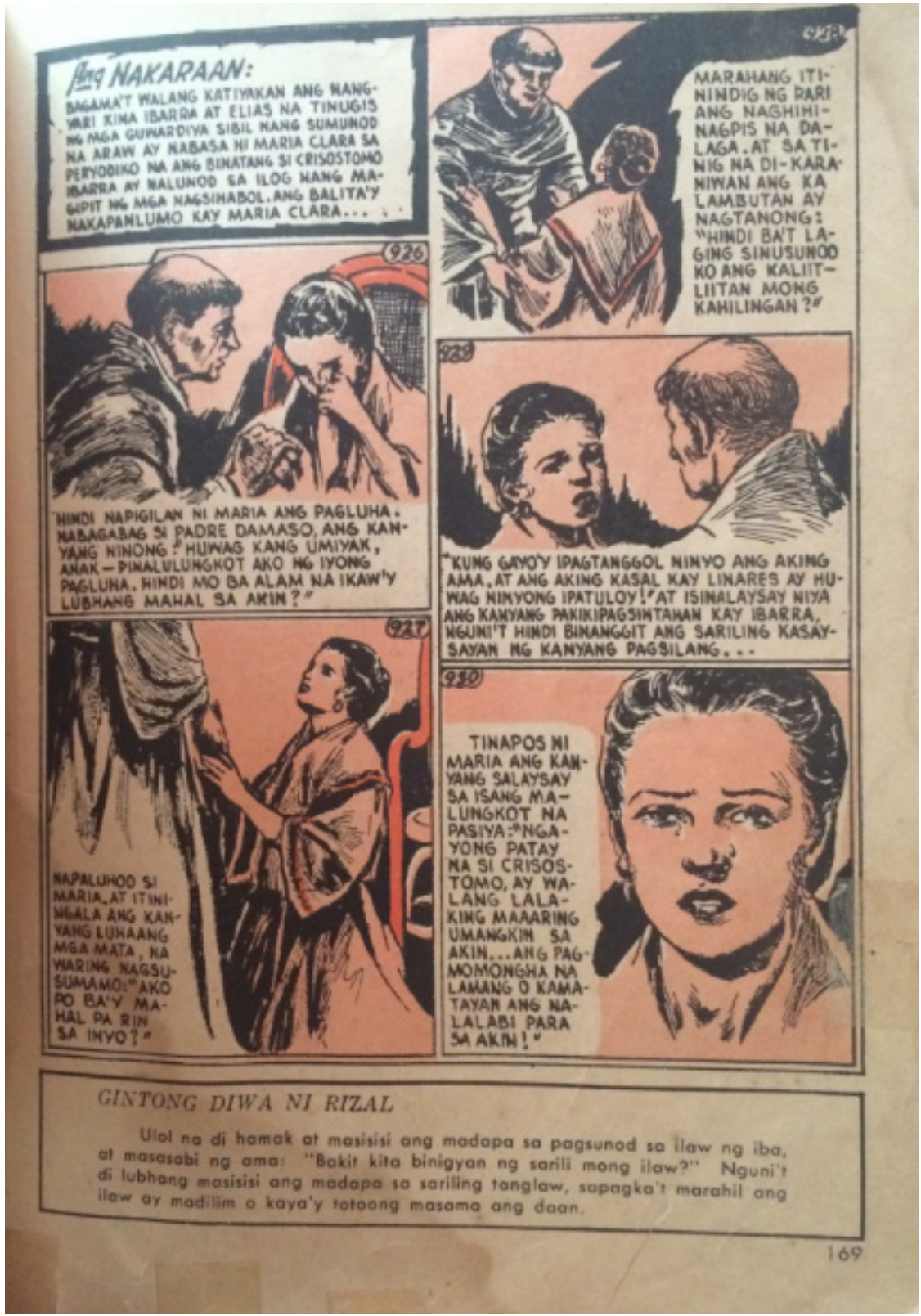

Panel 926-930 ng Nakalarawang Noli Me Tangere (1956). Dahil ang bungad ng tatlong pahinang ito ay “Ang Nakaraan," maipapalagay na tulad ng mga "graphic novel," kinulekta bilang isang libro ang dating seryalisadong NNMT. Mapapansin ding aktwal na laging nakatataas ang bumbunan ni Damaso kay Maria Clara. 


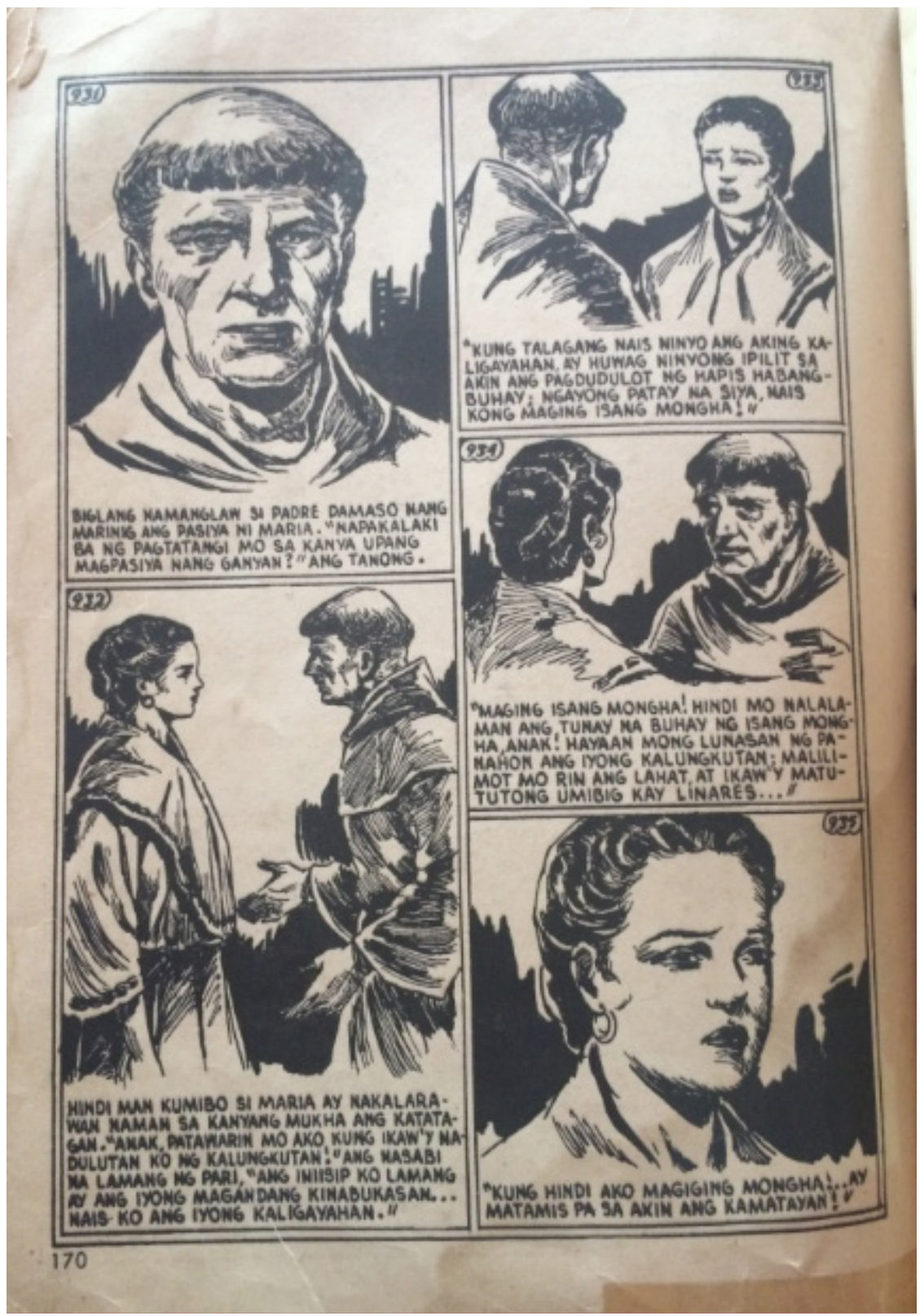

Panel 931-935 ng NNMT. Sa buong pagkakalatag o layout ng pahina, dominante pa rin ang prayle; sa indibidwal na mga panel, nakatataas pa rin at halos naaabot ng bumbunan ang kisame ng panel. Tulad sa nakaraang pahina, hindi na gumagamit dito ng "speech balloon," hindi tulad sa unang mga isyu o kabanata ng NNMT (na wala sa apendiseng ito).

Kritika Kultura 30 (2018): 521-525 


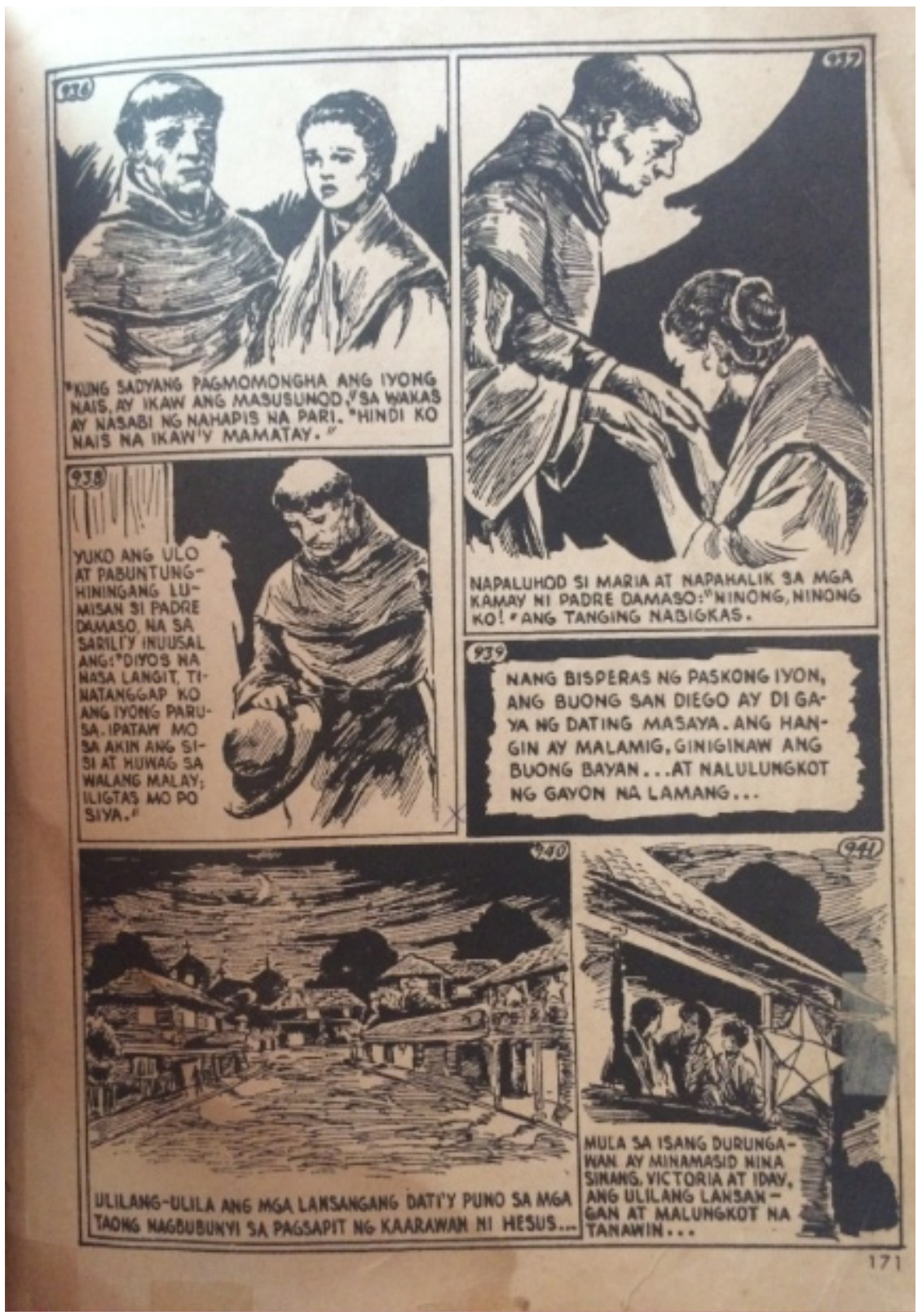

Panel 936-941. Sa puntong ito, halata namang kakatwa rin ang pagkakalatag ng panel, kaya kinakailangang idikta ang pagkakasunud-sunod ng pagbabasa ng mga ito. Sa mga komiks, may natural na pagbabasang inverted "S" o kaya'y simpleng "Z" ang direksyon: mula taas pababa, mula kaliwa pakanan. Ninong si Damaso rito, ayon sa pananalita ni Maria Clara. Tahimik ang nagsasalaysay sa pagka-Ama ng prayle. Nagkaiba rin ng "mood" itong mga pahina sa piraso ni Kampilan; iyak-tawa sa unang natutulak sa pag-iisip, pangungulila itong sa huli habang may tanglaw na parol. 


\section{Mga Sanggunian}

Abel, Jessica, and Matt Madden. "Foreword." The Best American Comics 2011, edited by Alison Bechdel, Houghton Mifflin, 2011, pp. vii-xii.

Acuna, Tilde. "Gallus Ignis, Angelus Novus, Lateralus." Materials, for Preposterity. DavaoToday. 28 Dec. 2017, http://davaotoday.com/main/todays-views/gallus-ignisangelus-novus-lateralus/. Accessed 4 Jan. 2018.

Anderson, Benedict. The Spectre of Comparisons : Nationalism, Southeast Asia, and the World. Ateneo de Manila UP, 2004.

Atkin, Albert. "Peirce's Theory of Signs." The Stanford Encyclopedia of Philosophy, 15 Nov. 2010, http://plato.stanford.edu/entries/peirce-semiotics/\#DivInt. Accessed 15 Nov. 2016.

Barbaza, Peñafrancia Raniela. "Wika at Identidad: Wikang Bikol bilang Lunan ng Bikolnon." Daluyan: Journal ng Wikang Filipino, vol. 20, 2014, pp. 73-98.

Beineke, Colin. Towards a Theory of Comic Book Adaptation. MA Thesis. University of Nebraska - Lincoln, 2011.

Bredehoft, Thomas. The Visible Text: Textual Production from Beowulf to Maus. Oxford UP, 2014.

Chandler, Daniel. Semiotics: The Basics. Routledge, 2002.

Cullum, Leo. "Literary Survey - 1954." Philippine Studies, vol. 3, no. 1, 1955, p. 90-96.

Danky, James, and Denis Kitchen. "Underground Classics: The Transformation of Comics into Comix, 1963-90." Underground Classics: The Transformation of Comics into Comix, edited by James Danky and Denis Kitchen, ComicArts, 2009, pp. 17-21.

David, Adam. "Rarefied Heights, Restoration, and the Rhythm Method: Some Analyses on the Mastery of Form of Francisco V. Coching's El Indio." Likhaan: The Journal of Contemporary Philippine Literature, vol. 4, 2010, pp. 120-130.

Dorfman, Ariel, and Armand Mattelart. How to Read Donald Duck: Imperialist Ideology in the Disney Comic. Translated by David Kunze. International General, 1981.

Eco, Umberto. A Theory of Semiotics. Indiana UP, 1976.

Ellis, Warren. "Foreword." The Art of Comics: A Philosophical Approach, edited by Aaron Meskin and Roy Cook, Blackwell, 2012, pp. xii-xiii.

Freire, Paulo. Pedagogy of the Oppressed. 1970. Translated by Myra Bergman Ramos. Continuum, 1984.

Guillermo, Alice. Images to Meaning: Essays on Philippine Art. Ateneo de Manila UP, 2001.

--. Protest / Revolutionary Art in the Philippines, 1970-1990. U of the Philippines P, 2001.

Hatfield, Charles. "Comix, Comic Shops and the Rise of Alternative Comics, Post 1968." Alternative Comics: An Emerging Literature, UP of Mississippi, 2005, pp. 3-31.

Hau, Caroline. Necessary Fictions: Philippine Literature and the Nation, 1946-1980. Ateneo de Manila UP, 2000.

--. On the Subject of Nation: Filipino Writings from the Margins, 1981-2004. Ateneo de Manila UP, 2004.

Hutcheon, Linda. A Theory of Adaptation. Routledge, 2006. 
Iniego Jr., Florentino. "Ang 'Ikalawang Paglilitis' kay Jose Rizal: Ang Pagpapasa ng Batas Republika 1425." Lagda: Journal ng U.P. Departamento ng Filipino at Panitikan ng Pilipinas, vol. 10, no. 1, 2015, pp. 151-168.

Jurilla, Patricia May. Tagalog Bestsellers of the Twentieth Century: A History of the Book in the Philippines. Ateneo de Manila UP, 2008.

Kampilan, Emiliana. "Clara, Join the Dark Side of the Force." Dead Balagtas, 20 Enero 2014, http://deadbalagtas.tumblr.com/post/73919954836/rizal-started-this-trope-i-swearanyway-ang-di. Accessed 25 Nob. 2016.

Kukkonen, Karin. Studying Comics and Graphic Novels. John Wiley \& Sons, 2013.

Lent, John. The First One Hundred Years of Philippine Komiks and Cartoons. Yonzon Associates, 2009.

Lumbera, Bienvenido. Writing the Nation: Pag-akda ng Bansa. U of the Philippines P, 2000.

McGowan, Todd. "The Barriers to a Critical Comedy." Crisis and Critique, vol. 1, no. 3, 2014., pp. 201-221.

Merrell, Floyd. "Charles Sanders Peirce's Concept of the Sign." The Routledge Companion to Semiotics and Linguistics, edited by Paul Cobley, Routledge, 2001, pp. 28-39.

Meskin, Aaron, and Roy Cook. The Art of Comics: A Philosophical Approach. Blackwell, 2012.

Miodrag, Hannah. Comics and Language: Reimagining Critical Discourse on the Form. UP of Mississippi, 2013.

Mitchell, W.J.T. "Image X Text." The Future of Text and Image: Collected Essays on Literary and Visual Conjectures, edited by Lauren Walsh Ofra Amihay, Cambridge Scholars Publishing, 2012, pp. 1-14.

-.. Picture Theory: Essays on Verbal and Visual Representation. U of Chicago P, 1994.

--. What Do Pictures Want?: The Lives and Loves of Images. U of Chicago P, 2005.

Mojares, Resil. Brains of the Nation: Pedro Paterno, TH Pardo de Tavera, Isabelo de los Reyes and the Producton of Modern Knowledge. Ateneo De Manila UP, 2006.

Peirce, Charles Sanders. "Logic as Semiotic: The Theory of Signs." Philosophical Writings of Peirce, edited by Justus Buchler, Dover, 1955, pp. 98-119.

Potts, Alex. "Signs." Critical Terms for Art History, edited by Robert Nelson and Richard Shiff, U of Chicago P, 1996, pp. 20-34.

Reyes, Soledad. A Dark Tinge to the World: Selected Essays (1987-2005). U of the Philippines P, 2005.

Rizal, Jose. Nakalarawang Noli Me Tangere. Edited by Clodualdo del Mundo and Pedrito Reyes. Capitol Publishing House, 1956.

Rosenkranz, Patrick. "The Limited Legacy of Underground Comix." Underground Classics: The Transformation of Comics into Comix, edited by James Danky and Denis Kitchen, Abrams ComicArt, 2009, pp. 23-30.

Roxas, Cynthia, and Joaquin Arevalo. A History of Komiks of the Philippines and Other Countries. Islas Filipinas Publishing, 1985.

Sabin, Roger. Adult Comics: An Introduction. Routledge, 1993.

Sendaydiego, Henry. “The Devil in a New Look." Varsitarian, 20 Dec 1951, pp. 24. Reprinted in Philippine Comic Magazine, pp. 54-58. 
Thomas, Megan. Orientalists, Propagandists and Ilustrados: Filipino Scholarship and the End of Spanish Colonialism. Anvil, 2016.

Tiongson, Nicanor. "Ang Pusong sa Dulang Tagalog: Panimulang Pag-aaral." Centennial Lecture Series: Memories, Visions, and Scholarship and Other Essays, University of the Philippines - Center for Ingrative Studies, 2001, pp. 305-338.

Uidihir, Christy Mag. "Comics and Collective Authorship." The Art of Comics: A Philosophical Approach, edited by Aaron Meskin and Roy Cook, Blackwell, 2012, pp. 47-67.

Valiente, Randy, and Fermin Salvador. Komiks sa Paningin ng mga Tagakomiks. Central Books Supply, Inc., 2007. 